\title{
Broken-symmetry states and divergent resistance in suspended bilayer graphene
}

\author{
Benjamin E. Feldman, Jens Martin and Amir Yacoby *
}

\begin{abstract}
Mono- and bilayer graphene have generated tremendous excitement owing to their unique and potentially useful electronic properties ${ }^{1}$. Suspending single-layer graphene flakes above the substrate ${ }^{2,3}$ has been shown to greatly improve sample quality, yielding high-mobility devices with little charge inhomogeneity. Here we report the fabrication of suspended bilayer graphene devices with very little disorder. We observe quantum Hall states that are fully quantized at a magnetic field of $0.2 \mathrm{~T}$, as well as broken-symmetry states at intermediate filling factors $v=0, \pm 1, \pm 2$ and \pm 3 . In the $v=0$ state, the devices show extremely high magnetoresistance that scales as magnetic field divided by temperature. This resistance is predominantly affected by the perpendicular component of the applied field, and the extracted energy gap is significantly larger than expected for Zeeman splitting. These findings indicate that the broken-symmetry states arise from manybody interactions and underscore the important part that Coulomb interactions play in bilayer graphene.
\end{abstract}

The linear dispersion of graphene near its Fermi energy gives rise to low-energy excitations that behave as massless Dirac fermions ${ }^{1}$. These quasiparticles show an anomalous integer quantum Hall effect $^{4,5}$, in which the Hall conductivity is quantized at values of $\sigma_{x y}=v e^{2} / h$ for filling factors $v=4(N+1 / 2)$. Here, $N$ is an integer, $e$ is the electron charge, $h$ is Planck's constant and the factor of four is due to spin and valley degeneracy. Recent measurements ${ }^{6,7}$ of graphene monolayers in high magnetic field $B$ have revealed more broken-symmetry quantum Hall states at $\nu=0$, \pm 1 and \pm 4 , which have been proposed to arise owing to quantum Hall ferromagnetism ${ }^{8,9}$ or the formation of excitonic energy gaps $^{10,11}$. The $v=0$ state has received particular attention owing to contradictory experimental observations. Some samples show large magnetoresistance of $\sim 10^{5}-10^{7} \Omega$ near the charge-neutrality point ${ }^{12-15}$, and this behaviour has been ascribed to the opening of a spin gap ${ }^{12}$, the approach of a field-induced Kosterlitz-Thouless transition to an insulating state ${ }^{13,14}$ or the formation of a collective insulator ${ }^{15}$. Others, however, report ${ }^{6,7,16}$ resistance of only $\sim 10^{4} \Omega$, and attribute their findings to the existence of spin-polarized counterpropagating edge modes ${ }^{6,16}$.

Although experimental investigations of broken-symmetry quantum Hall states have so far focused only on graphene monolayers, recent theoretical studies have investigated excitonic condensation $^{17}$ and quantum Hall ferromagnetism ${ }^{18}$ in bilayer graphene and the resultant ground states at intermediate filling factors ${ }^{19}$. The physics is richer in bilayers owing to an extra twofold orbital degeneracy in the Landau-level (LL) spectrum ${ }^{20}$, which leads to an eightfold-degenerate LL at zero energy and a corresponding step of $8 e^{2} / h$ in $\sigma_{x y}$. It has been shown both theoretically ${ }^{21}$ and experimentally ${ }^{22,23}$ that a potential difference between the two layers opens an energy gap, leading to a plateau in $\sigma_{x y}$ at $v=0$, but no other broken-symmetry states have been observed. Here we report the fabrication of high-quality suspended bilayer graphene devices (Fig. 1a,b) that show full splitting of the eightfold-degenerate zeroenergy LL. The $v=0$ state emerges at $B \approx 0.1 \mathrm{~T}$ and is characterized by an extremely large resistance that increases exponentially with the perpendicular component of $B$. The $|\nu|=2$ states emerge at $B=0.7 \mathrm{~T}$, and all symmetries are broken for $B \geq 3 \mathrm{~T}$.

We focus first on the behaviour of our samples in zero magnetic field. Figure 1c shows the resistivity $\rho$ of two suspended bilayers as a function of carrier density $n$. Each sample shows a sharp peak in $\rho$ with a full-width at half-maximum of the order of $10^{10} \mathrm{~cm}^{-2}$, comparable to that of suspended monolayer devices ${ }^{2,3}$ and an order of magnitude smaller than that of unsuspended bilayers $^{24}$. In all samples, the peak lies close to zero back-gate voltage $\left(\left|V_{\text {peak }}\right|<0.5 \mathrm{~V}\right)$, indicating that there is little extrinsic doping in our devices. As a measure of sample cleanliness, we can estimate the magnitude of carrier-density fluctuations $\delta n$ on the basis of the carrier-density dependence of the conductivity $\sigma(n)$, shown in Fig. 1d. Near the charge-neutrality point, local variations in potential lead to the formation of electron-hole puddles ${ }^{25}$, and $\sigma(n)$ is expected ${ }^{26}$ to remain constant in this regime because $|n|<\delta n$. In our suspended bilayers, $\delta n$ is typically of the order of $10^{10} \mathrm{~cm}^{-2}$, and it reaches as low as $10^{9} \mathrm{~cm}^{-2}$ in sample $\mathrm{S} 3$.

The temperature dependence of the minimum conductivity $\sigma_{\min }$ (Fig. 1e) provides a second method to estimate $\delta n$. At low temperatures, $\sigma_{\min }$ is dominated by transport through the electron-hole puddles rather than thermal effects, so we expect strong temperature dependence only for $k_{\mathrm{B}} T>E_{\text {pud }}$, where $k_{\mathrm{B}}$ is Boltzmann's constant and $E_{\text {pud }}$ is the typical magnitude of the screened potential fluctuations responsible for electron-hole puddles. For bilayer graphene, we can estimate $E_{\text {pud }} \approx h^{2} \delta n / 8 \pi m^{*}$, where $m^{*} \approx 0.033 m_{\mathrm{e}}$ is the effective mass in bilayer graphen ${ }^{26}$ ( $m_{\mathrm{e}}$ is the electron mass). In sample $\mathrm{S} 3, \sigma_{\min }$ shows temperature dependence down to $450 \mathrm{mK}$, providing an upper bound of $\delta n<10^{9} \mathrm{~cm}^{-2}$. In contrast, $\sigma_{\min }$ saturates at approximately $2 \mathrm{~K}$ in sample $\mathrm{S} 4$, corresponding to $\delta n \approx 5 \times 10^{9} \mathrm{~cm}^{-2}$. Both estimates are consistent with the estimate of disorder obtained from $\sigma(n)$. In both samples, $\sigma_{\min }$ at $450 \mathrm{mK}$ is a few times the conductance quantum, in good agreement with theoretical predictions for its intrinsic limit ${ }^{27,28}$.

In contrast to the typically reported linear behaviour in bilayer graphene, $\sigma(n)$ is sublinear in suspended samples (Fig. 1f). If we assume mobility $\mu=(1 / e) \mathrm{d} \sigma / \mathrm{d} n$, then $\mu$ typically ranges from 10,000 to $15,000 \mathrm{~cm}^{2} \mathrm{~V}^{-1} \mathrm{~s}^{-1}$ in our suspended bilayers at carrier densities of $2-3 \times 10^{11} \mathrm{~cm}^{-2}$. These numbers represent a modest improvement of approximately a factor of two over unsuspended bilayers, but it remains unclear why the mobility is this low given the indications of sample quality discussed above, the low magnetic field at which we observe quantum Hall plateaus and the high mobilities observed in suspended monolayers ${ }^{2,3}$. It is predicted ${ }^{26}$ that the mobility of bilayer graphene should be more than an order of magnitude smaller than that of monolayer graphene. 


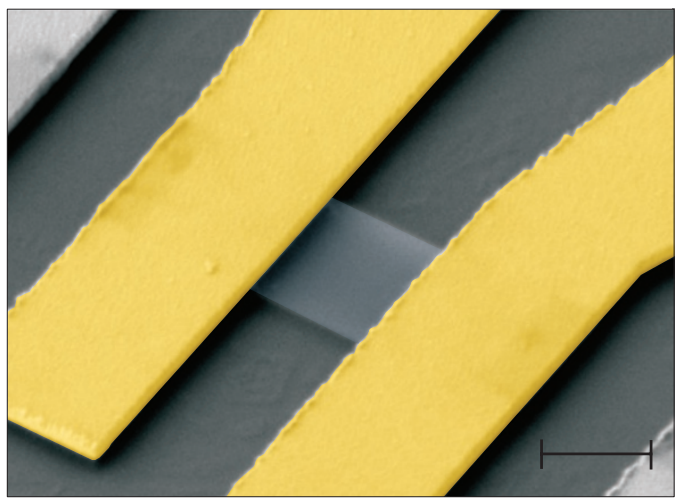

c
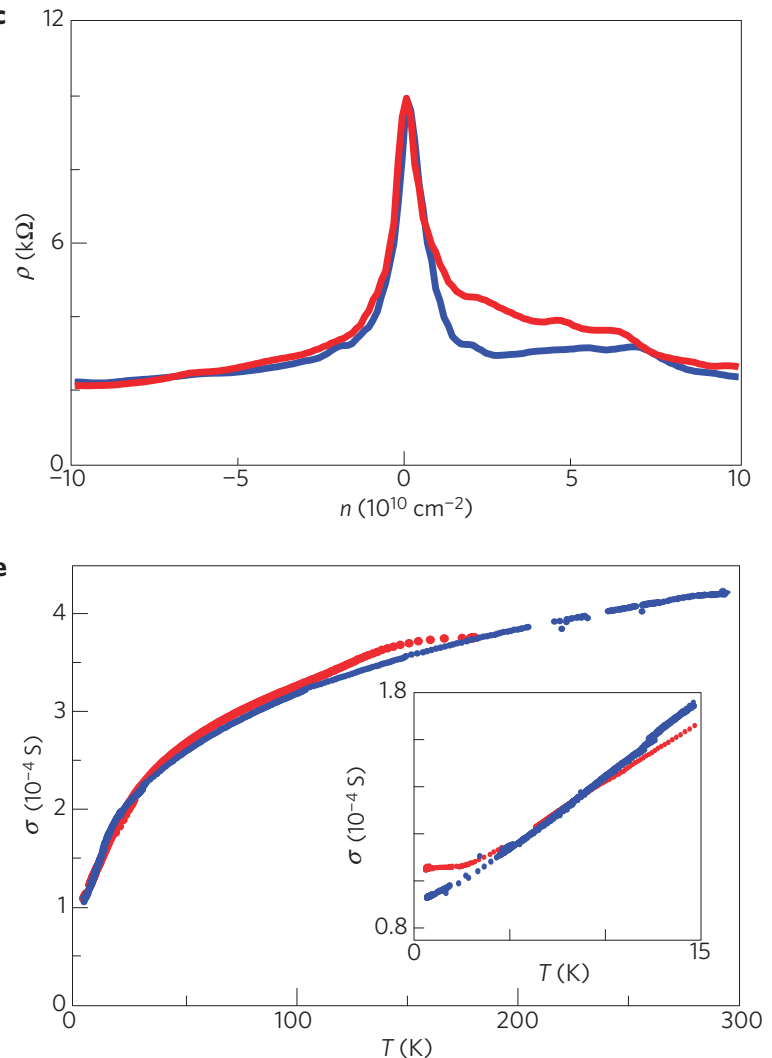

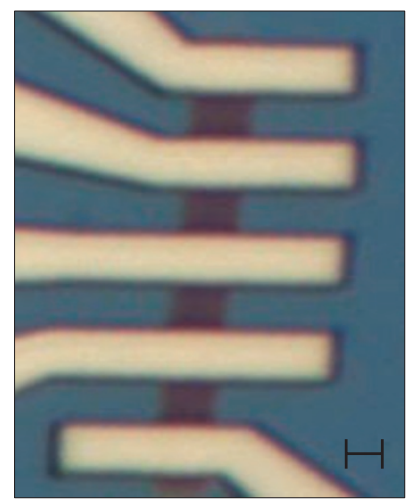

d 10

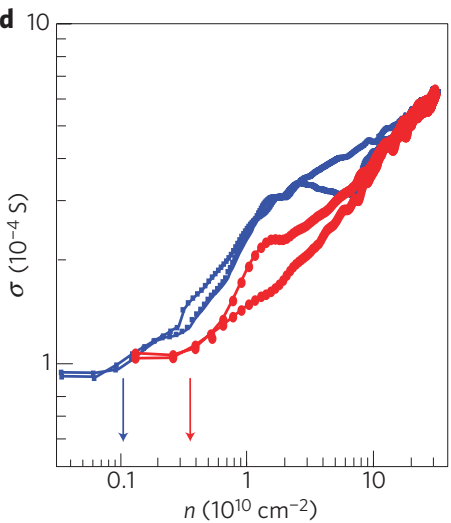

f

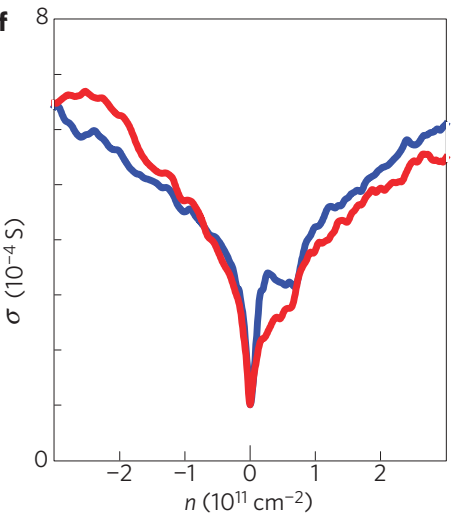

Figure 1 | Characterization of suspended bilayer samples S3 (blue) and S4 (red) at zero magnetic field. a, False-colour scanning electron micrograph of a typical suspended bilayer graphene flake. The scale bar is $1 \mu \mathrm{m}$. b. Optical microscope image of several two-terminal suspended bilayer samples in series. The scale bar is $1 \mu \mathrm{m}$. c. Two-terminal resistivity $\rho$ as a function of carrier density $n$. Both samples show a pronounced peak in $\rho$ with full-width at half-maximum of $1.5 \times 10^{10} \mathrm{~cm}^{-2}$ and $2 \times 10^{10} \mathrm{~cm}^{-2}$, respectively, at temperature $T=450 \mathrm{mK}$. d, Electron and hole branches of the conductivity $\sigma$ at $T=450 \mathrm{mK}$. The width of the plateau in $\sigma$, marked by the arrows, indicates the magnitude of carrier-density fluctuations due to disorder, estimated to be $10^{9} \mathrm{~cm}^{-2}$ in sample S3 and $4 \times 10^{9} \mathrm{~cm}^{-2}$ in sample S4. e, Temperature dependence of the minimal conductivity $\sigma_{\mathrm{min}}$. Inset: Zoom-in on the low-temperature behaviour. For sample $\mathrm{S} 4$, disorder causes $\sigma_{\mathrm{min}}$ to saturate for $T<2 \mathrm{~K}$. The decrease of $\sigma_{\min }$ for sample $\mathrm{S} 3$ down to $450 \mathrm{mK}$ indicates that it is cleaner, consistent with the findings in $\mathbf{c}$ and $\mathbf{d}$. $\mathbf{f}$, Conductivity at $T=450 \mathrm{mK}$. For $n>2 \times 10^{11} \mathrm{~cm}^{-2}$, the mobility is about $7,500 \mathrm{~cm}^{2} \mathrm{~V}^{-1} \mathrm{~s}^{-1}$. The pronounced dip in the conductivity at very low densities may be enhanced by a disorder-induced gap.

This discrepancy was not observed in unsuspended samples ${ }^{24}$, but mobility in such samples may be limited by disorder associated with the substrate. It is also worthwhile to comment on the possibility that the sharp dip in conductivity at low $n$ is enhanced by a small energy gap that opens owing to disorder-induced differences in carrier density between the top and bottom layers of the flake ${ }^{18}$. Differences in density of a few times $10^{9} \mathrm{~cm}^{-2}$ would lead to an energy gap ${ }^{23}$ of approximately $0.3 \mathrm{meV}$.

We next discuss the magnetic-field-dependent behaviour of our samples. Figure $2 \mathrm{a}, \mathrm{b}$ shows the conductance of sample S1 as a function of magnetic field and carrier density $G(n, B)$, and Fig. 2c highlights traces of $G(n, B)$ at several representative magnetic fields. Our devices show the expected quantum Hall conductance plateaus at $4 m e^{2} / h$ for bilayer graphene, corresponding to filling factors $v= \pm 4 m$. Full quantization for $v= \pm 4$ occurs at very low $B$, indicative of the cleanliness of our devices. In sample $S 3$, the $v= \pm 4$ plateaus are fully quantized at $0.2 \mathrm{~T}$ (Fig. $2 \mathrm{~d}$, inset).

As well as the expected behaviour highlighted above, we observe quantum Hall plateaus corresponding to intermediate filling factors $v=0, \pm 1, \pm 2$ and \pm 3 . The $|v|=2(1)$ state becomes apparent at 
a

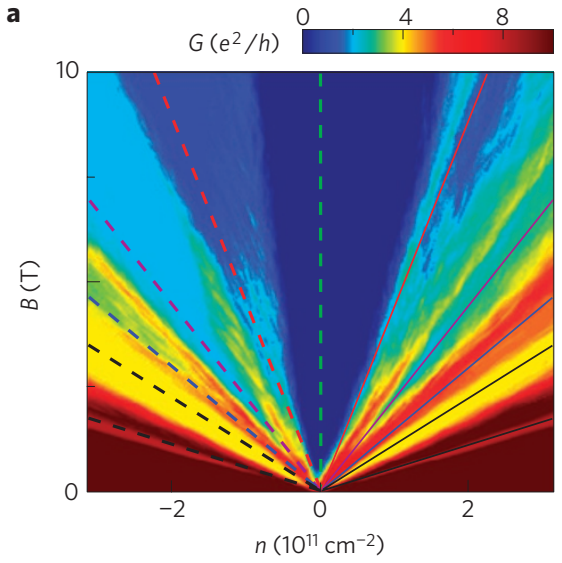

b

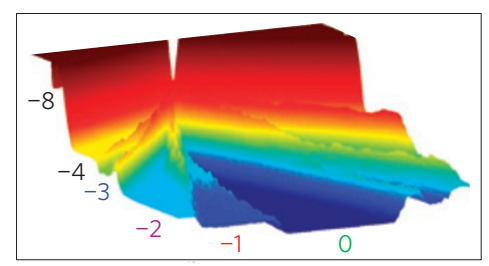

C

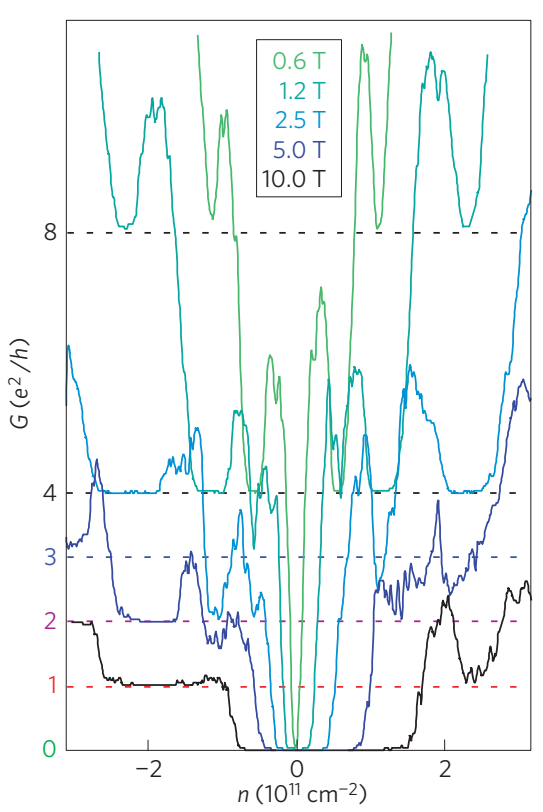

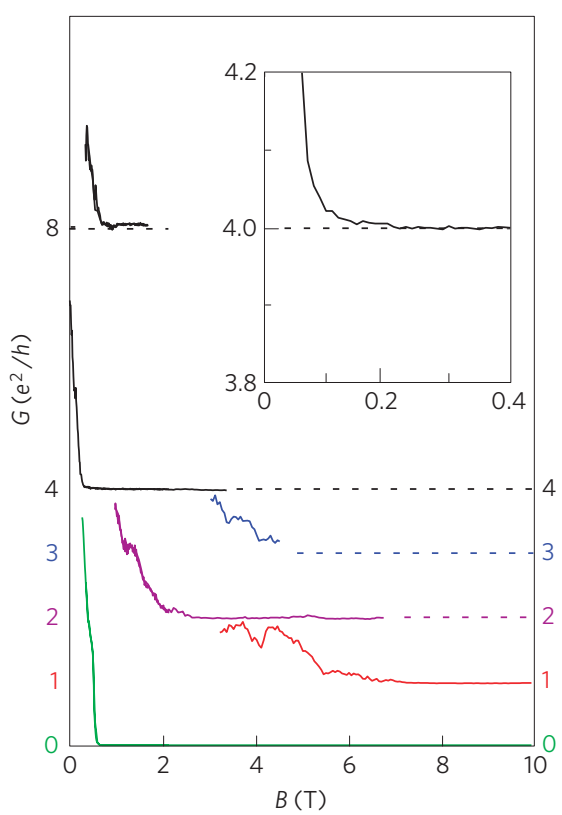

Figure 2 | Splitting of the eightfold-degenerate Landau level in suspended bilayers. a, Carrier-density and magnetic-field dependence of the two-terminal conductance $G(n, B)$ in sample $S 1$ at $T=100 \mathrm{mK}$. Lines indicate filling factors $|v|=8$ and 4 (black), 3 (blue), 2 (purple), 1 (red) and 0 (green). The conversion between back-gate voltage and density for each sample was calibrated using this type of measurement. $\mathbf{b}, 3 D$ rendering of $G(n, B)$ in sample S1. The numbers indicate filling factor. Broken-symmetry states at $v=0, \pm 1, \pm 2$ and \pm 3 are clearly visible. $\mathbf{c}$, Line traces of $G(n, B)$ at various magnetic fields. Quantum Hall plateaus associated with the broken-symmetry quantum Hall states are apparent. d, Conductance traces taken along the dotted lines in a. For sample S1, full quantization is observed at $B=0.4 \mathrm{~T}$ for $v=4, B=2.7 \mathrm{~T}$ for $v=2$ and $B=7.3 \mathrm{~T}$ for $v=1$. Inset: For sample $\mathrm{S} 3$, quantization of the $v=4$ state is reached for $B \approx 0.2 \mathrm{~T}$ at $T=450 \mathrm{mK}$.

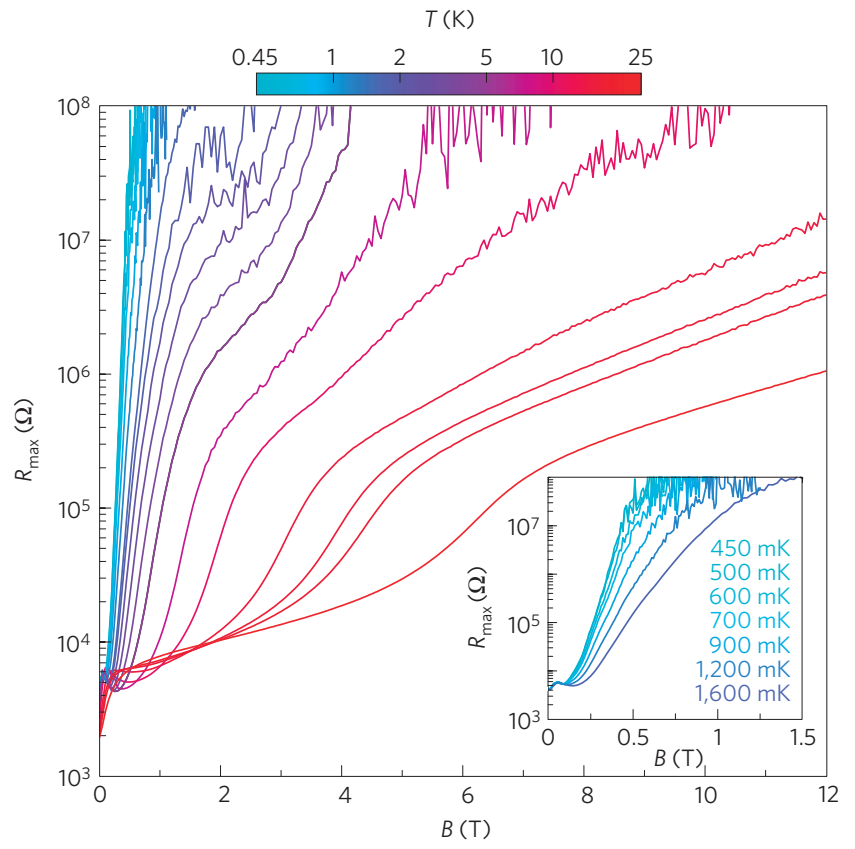

Figure 3 | Temperature and field dependence of the $v=0$ state. Maximum resistance of sample S3 at the charge-neutrality point as a function of magnetic field and temperature. Inset: Zoom-in on the low-temperature curves. We do not observe saturation of the resistance for temperatures down to $450 \mathrm{mK}$.

0.7 (2.7) $\mathrm{T}$, and fully develops into a conductance plateau of $2 e^{2} / h$ $\left(e^{2} / h\right)$ at $2.7(7.3) \mathrm{T}$ on the hole side (Fig. 2a,d). The $|v|=3$ state emerges at a similar magnetic field to the $|\nu|=1$ state, but leaves the experimentally accessible regime before it is fully quantized.
Near the charge-neutrality point, a $v=0$ state with a very large resistance that increases exponentially with $B$ emerges at $B \approx 0.1 \mathrm{~T}$. Measurements of Hall bar devices show a corresponding plateau at $\sigma_{x y}=0$ and rule out the possibility that the large resistance arises from contact resistance between the graphene and the electrical leads. We focus, however, on two-terminal devices because they are more homogeneous (see Supplementary Information).

The appearance of quantum Hall states at $v=0, \pm 1, \pm 2$ and \pm 3 indicates that the eightfold degeneracy of the zero-energy LL in bilayer graphene is completely lifted in our samples. The magnetic field at which these effects emerge is over an order of magnitude smaller than has been reported for monolayers $6,7,12-16$. Broken-symmetry states could arise from several causes, including spin splitting due to the Zeeman effect ${ }^{12}$, strain-induced lifting of valley degeneracy ${ }^{29}$, the opening of an energy gap due to a potential difference between the two layers or Coulomb interactions ${ }^{17,18}$. In our samples, the proximity of $V_{\text {peak }}$ to zero back-gate voltage makes it unlikely that we observe an energy gap due to chemical doping ${ }^{23}$. It has recently been shown ${ }^{30}$ that large-scale ripples appear in suspended graphene membranes when they are cooled from 600 to $300 \mathrm{~K}$, but room-temperature scanning electron micrographs of our suspended flakes do not show prominent corrugations (Fig. 1a). The interaction energy due to Coulomb effects in bilayer graphene is expected to be two orders of magnitude stronger than spin splitting caused by the Zeeman effect ${ }^{17,18}$, so the observed broken-symmetry states are unlikely to be associated with Zeeman splitting. We therefore tentatively attribute the symmetry breaking to Coulomb interactions. The order in which broken-symmetry states emerge in our samples is indeed consistent with the expectations of Barlas et al., who predict ${ }^{18}$ the largest energy gap for a spinpolarized state at $v=0$, followed by spin- and valley-polarized states at $|v|=2$ and finally spin-, valley- and LL-index-polarized states at $|v|=1$ and $|\nu|=3$. 


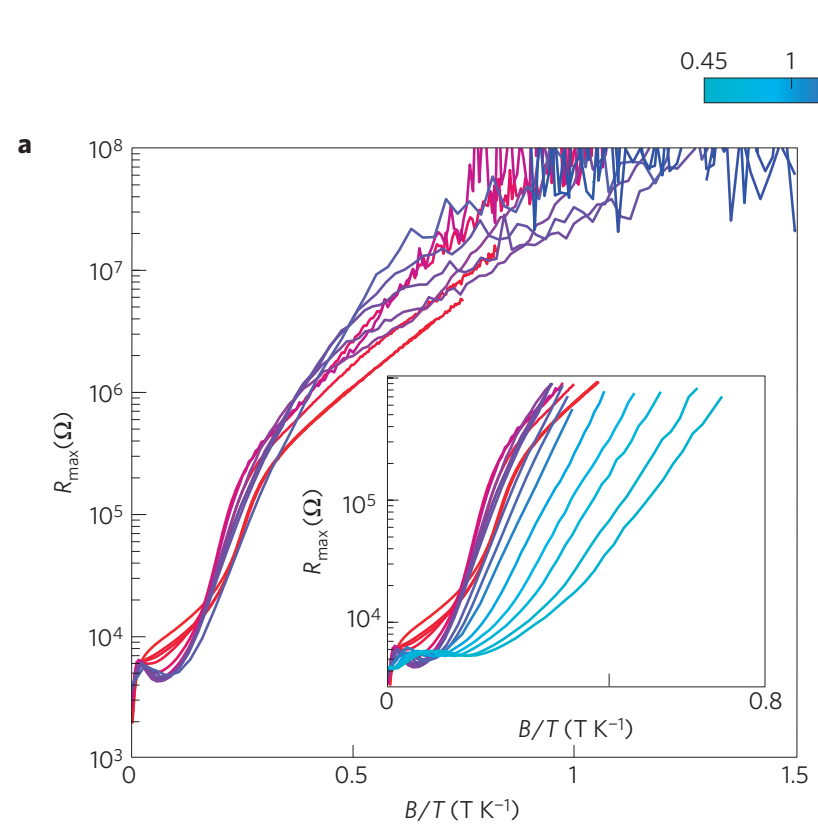

$T(\mathrm{~K})$

$2 \quad 5 \quad 10 \quad 25$

C

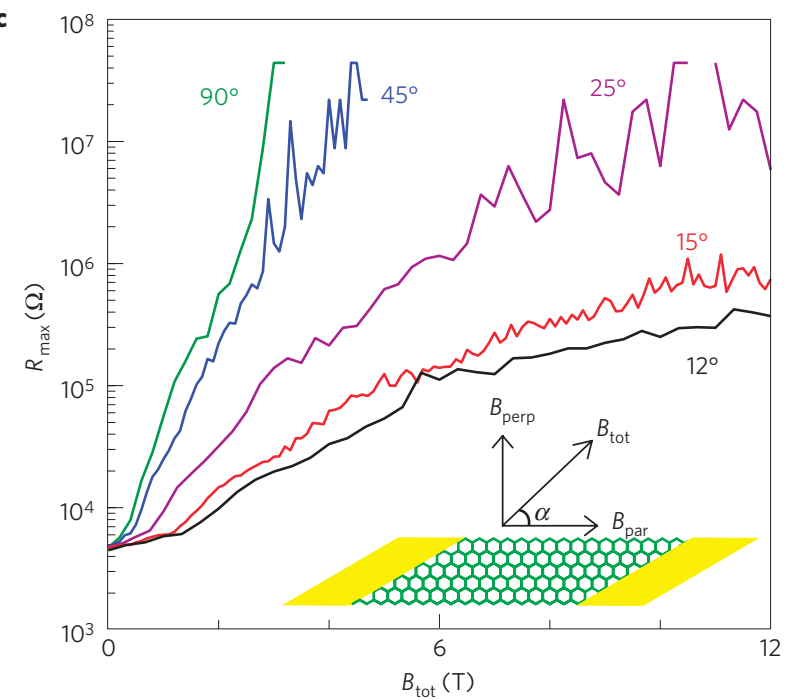

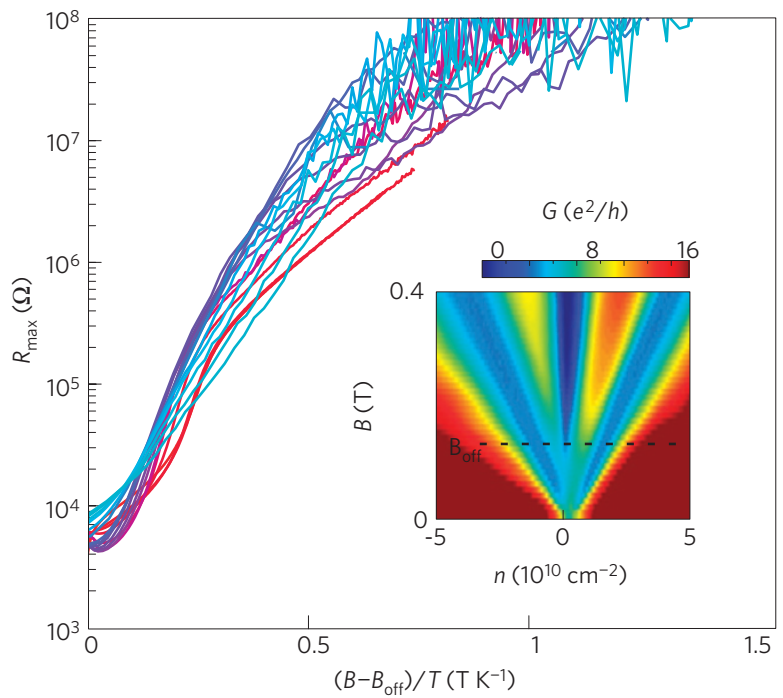

d

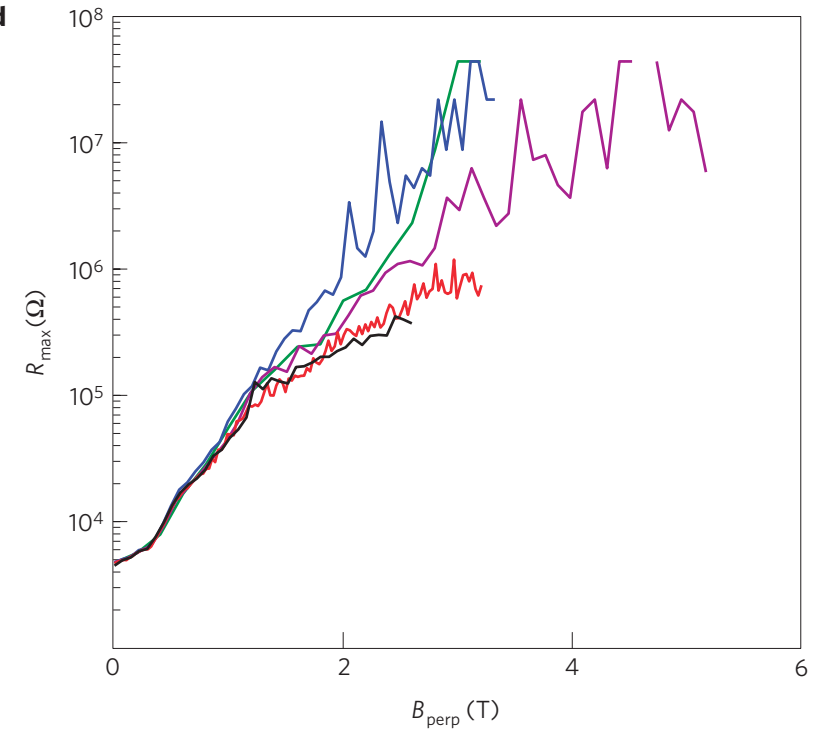

Figure 4 | Scaling of the maximum resistance in the $\boldsymbol{v}=\mathbf{0}$ state. $\mathbf{a}, R_{\max }(B, T)$ of sample $S 3$ plotted versus $B / T$. The data collapse onto one curve for temperatures $T>1.9 \mathrm{~K}$. Inset: $B / T$ scaling does not succeed for $T<1.9 \mathrm{~K} . \mathbf{b}, R_{\max }(B, T)$ versus $\left(B-B_{\text {off }}\right) / T$. All data collapse using $B_{\text {off }}=0.14 T$, which arises owing to disorder in the bilayer. Inset: Two-terminal conductance as a function of density and magnetic field. $B_{\text {off }}$ coincides with quantization of the $v= \pm 4$ plateaus and the emergence of the $v=0$ state. $\mathbf{c}, R_{\max }(B, T)$ of sample S2 as a function of total applied magnetic field for several angles $\alpha$ between sample and field. Inset: Schematic showing the relative orientation between field and sample. $\mathbf{d}, R_{\max }(B, T)$ as a function of the perpendicular component of the magnetic field for the same angles as in $\mathbf{c}$. The resistance depends primarily on $B_{\text {perp }}$, contradicting the expected behaviour for a Zeeman gap.

We now discuss in more detail the large magnetoresistance of the $v=0$ state. Figure 3 shows the maximum resistance of sample S3 in a small carrier-density range around the charge-neutrality point as a function of magnetic field and temperature, $R_{\max }(B, T)$ at various temperatures between $450 \mathrm{mK}$ and $24.5 \mathrm{~K}$ (see also the Supplementary Information). $R_{\max }(B, T)$ increases by more than four orders of magnitude to $10^{8} \Omega$ (the de facto limit of our measurement capabilities) within a few Tesla for $T<5 \mathrm{~K}$. This increase is significantly steeper than in monolayers, where the reported ${ }^{14}$ resistance reached only $40 \mathrm{M} \Omega$ at $30 \mathrm{~T}$. Our data do not fit a Kosterlitz-Thouless-type transition, nor do the flakes show activated behaviour over the full temperature range of our measurements.

One of the main findings of this report is that $R_{\max }(B, T)$ scales as $B / T$, as plotted in Fig. 4a. For $T \geq 1.9 \mathrm{~K}$, the data collapse rather nicely onto one curve. At lower temperatures, $R_{\max }(B, T)$ continues to increase with decreasing $T$, but it does not do so as quickly as expected for $B / T$ dependence (Fig. $4 \mathrm{a}$, inset). This can be explained if we assume that the LLs are broadened by disorder. In such a scenario, a constant offset in magnetic field $B_{\text {off }}$ is needed to resolve distinct quantum Hall states. Using $B_{\text {off }}=0.14 \mathrm{~T}$, in reasonable agreement with the field at which the $|\nu|=4$ states become fully quantized and the $v=0$ resistance begins to diverge (Fig. $4 \mathrm{~b}$, inset), the $R_{\max }(B, T)$ data collapse onto one curve for the entire temperature range when plotted against $\left(B-B_{\text {off }}\right) / T$ (Fig. $\left.4 \mathrm{~b}\right)$.

We infer that an energy gap $\Delta \sim 0.3-0.9(B[\mathrm{~T}]) \mathrm{meV}$ develops in an applied magnetic field. The gap is several times larger than expected for Zeeman splitting, and tilted-field experiments provide further evidence that the broken-symmetry states probably arise from many-body effects rather than Zeeman splitting. $R_{\max }(B, T)$ is primarily dictated by the perpendicular component of field $B_{\text {perp }}$ 
(Fig. 4c,d), in stark disagreement with the behaviour expected for a Zeeman gap. Moreover, at fixed $B_{\text {perp }}$, an increase in the parallel component of the field reduces $R_{\max }(B, T)$ (Fig. $4 \mathrm{~d}$ ), indicating that the low-energy excitations of the $v=0$ state are not skyrmionic spin flip in nature $e^{19}$. The linear dependence of $\Delta$ on $B$ is qualitatively different from what is expected for quantum Hall ferromagnetism and magnetic catalysis, which both predict ${ }^{17,18,31,32}$ a gap that scales as $B^{1 / 2}$. It is worth noting, however, that early studies ${ }^{33}$ of the exchange-enhanced spin gap at $v=1$ in GaAs samples also showed an energy gap that was linear in $B$. Coulomb interactions are predicted $^{17,18}$ to generate $\Delta \sim 100 \mathrm{meV}$ for magnetic fields of a few Tesla, far larger than we observe, but this discrepancy is probably due to disorder in our samples.

\section{Methods}

Suspended bilayer graphene devices are fabricated using a method similar to that described in ref. 2. Briefly, mechanical exfoliation of highly oriented pyrolytic graphite (grade ZYA, SPI Supplies) is used to deposit few-layer graphene flakes on a $\mathrm{Si}$ substrate coated with a $300 \mathrm{~nm}$ layer of $\mathrm{SiO}_{2}$. Deposition is carried out at $180^{\circ} \mathrm{C}$ to minimize the amount of water present on the substrate. Bilayer flakes are identified using an optical microscope, on the basis of contrast between the flake and the surrounding substrate. Electrical leads are then patterned using electron-beam lithography, followed by thermal evaporation of $3 \mathrm{~nm}$ of $\mathrm{Cr}$ and $100 \mathrm{~nm}$ of Au, and subsequent liftoff in warm acetone. The entire substrate is then immersed in 5:1 buffered oxide etch for $90 \mathrm{~s}$, which etches approximately $40 \%$ of the $\mathrm{SiO}_{2}$, including the area under the graphene ${ }^{2}$, but not the area under the metal contacts, which extend across the entire width of the flake to improve structural integrity. Samples are quickly transferred to methanol and dried using a critical-point dryer. Finished samples are transferred to the measurement system as quickly as possible, and are typically used without further cleaning or current annealing. Electronic transport measurements have been made on multiple samples, using standard a.c. lock-in techniques with excitation voltages below $100 \mu \mathrm{V}$, in either an ultrahigh-vacuum He- 3 cryostat or a dilution refrigerator. The Si substrate serves as a global back gate, which is used to vary the carrier density in the bilayer. Back-gate voltage is limited to $\left|V_{\mathrm{bg}}\right|<10 \mathrm{~V}$ to avoid structural collapse of suspended devices.

Received 15 June 2009; accepted 26 August 2009; published online 27 September 2009

\section{References}

1. Castro Neto, A. H., Guinea, F., Peres, N. M. R., Novoselov, K. S. \& Geim, A. K. The electronic properties of graphene. Rev. Mod. Phys. 81, 109-162 (2009).

2. Bolotin, K. I. et al. Ultrahigh electron mobility in suspended graphene. Solid State Commun. 146, 351-355 (2008).

3. Du, X., Skachko, I., Barker, A. \& Andrei, E. Y. Approaching ballistic transport in suspended graphene. Nature Nanotech. 3, 491-495 (2008).

4. Novoselov, K. S. et al. Two-dimensional gas of massless Dirac fermions in graphene. Nature 438, 197-200 (2005).

5. Zhang, Y. B., Tan, Y. W., Stormer, H. L. \& Kim, P. Experimental observation of the quantum Hall effect and Berry's phase in graphene. Nature 438, 201-204 (2005).

6. Jiang, Z., Zhang, Y., Stormer, H. L. \& Kim, P. Quantum Hall states near the charge-neutral Dirac point in graphene. Phys. Rev. Lett. 99, 106802 (2007).

7. Zhang, Y. et al. Landau-level splitting in graphene in high magnetic fields. Phys. Rev. Lett. 96, 136806 (2006).

8. Nomura, K. \& MacDonald, A. H. Quantum Hall ferromagnetism in graphene. Phys. Rev. Lett. 96, 256602 (2006).

9. Alicea, J. \& Fisher, M. P. A. Graphene integer quantum Hall effect in the ferromagnetic and paramagnetic regimes. Phys. Rev. B 74, 075422 (2006).

10. Gusynin, V. P., Miransky, V. A., Sharapov, S. G. \& Shovkovy, I. A. Excitonic gap, phase transition, and quantum Hall effect in graphene. Phys. Rev. B 74, 195429 (2006).

11. Khveshchenko, D. V. Composite Dirac fermions in graphene. Phys. Rev. B 75, 153405 (2007).
12. Giesbers, A. J. M. et al. Gap opening in the zeroth Landau level of graphene. Preprint at <http://arxiv.org/abs/0904.0948vl > (2009).

13. Checkelsky, J. G., Li, L. \& Ong, N. P. Zero-energy state in graphene in a high magnetic field. Phys. Rev. Lett. 100, 206801 (2008).

14. Checkelsky, J. G., Li, L. \& Ong, N. P. Divergent resistance at the Dirac point in graphene: Evidence for a transition in a high magnetic field. Phys. Rev. B 79, 115434 (2009).

15. Zhang, L. et al. Breakdown of the $N=0$ quantum Hall state in graphene: Two insulating regimes. Preprint at <http://arxiv.org/abs/0904.1996v2 > (2009).

16. Abanin, D. A. et al. Dissipative quantum Hall effect in graphene near the Dirac point. Phys. Rev. Lett. 98, 196806 (2007).

17. Ezawa, M. Intrinsic Zeeman effect in graphene. J. Phys. Soc. Jpn 76, 097401 (2007).

18. Barlas, Y., Cote, R., Nomura, K. \& MacDonald, A. H. Intra-Landau-level cyclotron resonance in bilayer graphene. Phys. Rev. Lett. 101, 097601 (2008).

19. Abanin, D. A., Parameswaran, S. A. \& Sondhi, S. L. Charge 2e skyrmions in bilayer graphene. Phys. Rev. Lett. 103, 076802 (2009).

20. Novoselov, K. S. et al. Unconventional quantum Hall effect and Berry's phase of 2 pi in bilayer graphene. Nature Phys. 2, 177-180 (2006).

21. McCann, E. \& Fal'ko, V. I. Landau-level degeneracy and quantum Hall effect in a graphite bilayer. Phys. Rev. Lett. 96, 086805 (2006).

22. Oostinga, J. B., Heersche, H. B., Liu, X. L., Morpurgo, A. F. \& Vandersypen, L. M. K. Gate-induced insulating state in bilayer graphene devices. Nature Mater. 7, 151-157 (2008).

23. Castro, E. V. et al. Biased bilayer graphene: Semiconductor with a gap tunable by the electric field effect. Phys. Rev. Lett. 99, 216802 (2007).

24. Morozov, S. V. et al. Giant intrinsic carrier mobilities in graphene and its bilayer. Phys. Rev. Lett. 100, 016602 (2008).

25. Martin, J. et al. Observation of electron-hole puddles in graphene using a scanning single-electron transistor. Nature Phys. 4, 144-148 (2008).

26. Adam, S. \& Das Sarma, S. Boltzmann transport and residual conductivity in bilayer graphene. Phys. Rev. B 77, 115436 (2008).

27. Katsnelson, M. I. Minimal conductivity in bilayer graphene. Eur. Phys. J. B 52, 151-153 (2006).

28. Snyman, I. \& Beenakker, C. W. J. Ballistic transmission through a graphene bilayer. Phys. Rev. B 75, 045322 (2007).

29. Abanin, D. A., Lee, P. A. \& Levitov, L. S. Randomness-induced XY ordering in a graphene quantum Hall ferromagnet. Phys. Rev. Lett. 98, 156801 (2007).

30. Bao, W. et al. Controlled ripple texturing of suspended graphene and ultrathin graphite membranes. Nature Nanotech. 4, 562-566 (2009).

31. Khveshchenko, D. V. Magnetic-field-induced insulating behavior in highly oriented pyrolitic graphite. Phys. Rev. Lett. 87, 206401 (2001).

32. Gorbar, E. V., Gusynin, V. P., Miransky, V. A. \& Shovkovy, I. A. Magnetic field driven metal-insulator phase transition in planar systems. Phys. Rev. B 66, 045108 (2002).

33. Dolgopolov, V. T. et al. Direct measurements of the spin gap in the two-dimensional electron gas of AlGaAs-GaAs heterojunctions. Phys. Rev. Lett. 79, 729-732 (1997).

\section{Acknowledgements}

We would like to acknowledge discussions with L. S. Levitov, R. Nandkishore, D. A. Abanin, A. H. Castro Neto, A. H. MacDonald, M. S. Rudner and S. Sachdev. We acknowledge support from Harvard NSEC, the ONR MURI program and Harvard CNS, a member of the NNIN, which is supported by the NSF.

\section{Author contributions}

B.E.F. conceived and designed the experiments, fabricated samples, carried out the experiments and data analysis and wrote the paper. J.M. conceived and designed the experiments, carried out the experiments and data analysis and wrote the paper. A.Y conceived and designed the experiments, carried out data analysis and wrote the paper.

\section{Additional information}

Supplementary information accompanies this paper on www.nature.com/naturephysics. Reprints and permissions information is available online at http://npg.nature.com/ reprintsandpermissions. Correspondence and requests for materials should be addressed to A.Y. 